\title{
CONTINUATION AND REFLECTION OF SOLUTIONS OF PARTIAL DIFFERENTIAL EQUATIONS ${ }^{1}$
}

\author{
FRITZ JOHN
}

A solution of an ordinary differential equation can be continued as long as its graph stays in the domain, in which the equation is regular. On the other hand a solution of a partial differential equation can have a natural boundary interior to the domain of regularity of the equation. Let $R$ be a closed region and $S$ a portion of the boundary of $R$. Then $S$ is a natural boundary for a solution $u$ defined in $R$, if there exists no solution defined in a full neighbourhood of a point of $S$ which agrees with $u$ in $R$. Examples for the occurrence of such natural boundaries are well known from the theory of harmonic functions. Neither the equation nor the solution has to show any very singular behavior on approaching a natural boundary $S$.

Only in very special situations can one prove that every sufficiently regular solution known in a region $R$ can be continued across a portion $S$ of the boundary. This is e.g. the case for solutions of a single differential equation which is hyperbolic with respect to $S$. It is also the case for solutions of certain overdetermined systems of equations, like those associated with analytic functions of several complex variables; other systems with this property have been studied by S. Bochner [1].

In more general cases the only solutions for which one can prove continuibility are those satisfying suitable boundary conditions on $S$. The classical example is furnished by solutions $u(x, y)$ of the Laplace equation

$$
u_{x x}+u_{y y}=0
$$

defined for $y \geqq 0$ and satisfying the boundary condition

$$
u(x, 0)=0 \text {. }
$$

They can always be continued across $y=0$ by the formula

$$
u(x,-y)=-u(x, y) \text {. }
$$

An address delivered before the New York meeting of the Society on April 21, 1956, by invitation of the Committee to Select Hour Speakers for Eastern Sectional Meetings; received by the editors January 11, 1957.

1 A summary of the results of this paper appeared in Colloques internationaux $d u$ centre national de la recherche scientifique, vol. 71, Nancy, 1956. 
In recent years one has succeeded in extending this continuation theorem in various ways, involving either more general boundary conditions or more general differential equations. I mention the following generalizations. Thus a sufficiently regular solution of (1a) defined for $y \geqq 0$ can be continued across $y=0$ whenever it satisfies instead of (1a) a boundary condition of the form

$$
u_{y}=A\left(x, u, v, u_{x}\right)
$$

on $y=0$, where $A$ is an analytic function of its arguments and $v$ denotes the conjugate harmonic to $u$ (See H. Lewy [2], R. Gerber [3]). A solution of any linear elliptic equation of any order and in any number of dimensions which has vanishing Dirichlet data on a hyperplane can be continued across that plane, provided the equation has analytic coefficients. (See Morrey [5], Nirenberg and Morrey [4].) A solution of the equation of minimal surfaces that is analytic on an analytic boundary curve can be continued across that curve. (See Lewy [15].)

Continuation of solutions constitutes an important tool in finding an explicit solution of many problems of applied mathematics. It has been used by Helmholtz, Kirchhoff, Shiffman and others in determining motions of liquids possessing a free boundary. ${ }^{2}$ In these problems the continuation of the flow is not explicit as long as the location of the free boundary is not known; the free boundary and the flow are obtained simultaneously by also taking into account the boundary conditions on fixed boundaries and reducing the problem to one in conformal mapping. Explicit local continuation can be used, when the boundary conditions are sufficiently simple. This situation arises e.g. in the linearized theory of gravity waves, where one is led to consider solutions of the Laplace equation (1a), which satisfy a boundary condition of the form

$$
u_{\nu}+k u=0
$$

with constant $k$ on the $x$-axis. ${ }^{3}$ The continuation of solutions of more general second order equations with constant coefficients which satisfy boundary conditions of the type (2) on a plane has been studied by Diaz and Ludford [9].

A few cases in which solutions of higher order equations can be continued by an explicit reflection formula have been established. Poritsky [10] proved that a solution $u(x, y)$ of the bi-harmonic equation

$$
\Delta^{2} u=0
$$

${ }^{2}$ See Lamb [6], Ch. IV, Milne-Thomson [7].

${ }^{3}$ See Stoker [8]. 
which satisfies the boundary conditions

$$
u=u_{y}=0 \quad \text { for } y=0
$$

can be continued across the $x$-axis using the formula

$$
u(x,-y)=-u(x, y)+2 y u_{y}(x, y)-y^{2} \Delta u(x, y) .
$$

Analogous formulae have been obtained by Duffin $[11 ; 12]$ for the systems of equations describing static equilibrium of a 3-dimensional isotropic elastic body. Finally Huber [13] has generalized formula (3c) to the case of solutions of a poly-harmonic equation

$$
\Delta^{p} u\left(x_{1} \cdots, x_{n}\right)=0
$$

having vanishing Dirichlet data on a hyper-plane.

It seems desirable to have a more systematic study of the types of boundary conditions that guarantee existence of a continuation of the solution of a differential equation and also of the cases in which such continuation can be achieved by an explicit formula. Of course, such formulae can be expected only for very simple situations, say for linear equations with constant coefficients and for solutions satisfying linear homogeneous boundary conditions with constant coefficients on a plane boundary. I should like to present here some results in this direction for a rather simplified situation: The equation shall be linear and homogeneous and have constant coefficients, the boundary shall be plane, and the boundary conditions shall be of the type that the solution and a certain number of its derivatives vanish on the boundary plane. The results have been extended by R. I. Canavan to the most general equation with constant coefficients in his thesis on Necessary conditions for continuation and reflection principles for solutions of linear partial differential equations with constant coefficients, New York University, 1957.

There shall be $n+1$ independent variables $x_{0}, x_{1}, \cdots, x_{n}$. We write $x$ for the $n$-vector $\left(x_{1}, \cdots, x_{n}\right)$, and $u\left(x_{0}, x\right)$ for the function $u\left(x_{0}, x_{1}, \cdots, x_{n}\right)$. The differential equation shall be of the form

$$
P\left(\xi_{0}, \xi\right) u\left(x_{0}, x\right)=0
$$

where $P\left(\xi_{0}, \xi\right)$ is a form with constant complex coefficients of degree $N$ in

$$
\xi_{0}=\partial / \partial x_{0} \text { and } \xi=\left(\partial / \partial x_{1} \cdots, \partial / \partial x_{n}\right) .
$$

Moreover we make the assumption

$$
P(1,0)=1 \text {, }
$$


equivalent to noncharacteristic character of the plane $x_{0}=0$. The boundary condition shall be of the form

$$
\left(\xi_{0}^{k} u\right)_{x_{0}=0}=0 \quad \text { for } k=0, \cdots, s-1 .
$$

We shall show that in general if $s$ is too small there exist solutions of $(5 \mathrm{a}, \mathrm{c})$ which are in $C_{\infty}$ for $x_{0} \geqq 0$ and have the plane $x_{0}=0$ as natural boundary.

Solutions of partial differential equations with natural boundaries can be obtained in the form of series similar to those used for constructing analytic functions with natural boundaries. We consider the characteristic equation of equation (5a):

$$
P\left(\eta_{0}, \eta\right)=0 .
$$

For given $\eta=\left(\eta_{1}, \cdots, \eta_{n}\right)$ the $N$ roots $\eta_{0}$ of (6) may be denoted by $\lambda_{1}, \cdots, \lambda_{N}$. Assume now that for a certain real $\eta$ with $\eta \cdot \eta=1$ the roots $\eta_{0}$ of (6) satisfy the conditions

$$
\operatorname{Im} \lambda_{1} \geqq \operatorname{Im} \lambda_{2}, \cdots, \operatorname{Im} \lambda_{s+1} \geqq 0
$$

and

$$
\operatorname{Im} \lambda_{1}>0 .
$$

Let $f\left(x_{0}\right)$ be the solution of the ordinary differential equation

$$
\prod_{k=1}^{s+1}\left(\xi_{0}-i \lambda_{k}\right) f\left(x_{0}\right)=0
$$

with initial conditions

$$
\xi_{0}^{k} f\left(x_{0}\right)=\left\{\begin{array}{l}
0 \text { for } k=0, \cdots, s-1, \\
1 \text { for } k=s
\end{array}\right.
$$

for $x_{0}=0$. Then, because of (7a), $f\left(x_{0}\right)$ and any of its derivatives become infinite at most like a power of $x_{0}$ for $x_{0} \rightarrow+\infty$. The function

$$
e^{i \eta \cdot x} f\left(x_{0}\right)
$$

is a solution of $(5 a, c)$. It follows that

$$
u\left(x_{0}, x\right)=\sum_{j=0}^{\infty} e^{-j^{2}} \exp \left(i 2^{i} \eta \cdot x\right) f\left(2^{j} x_{0}\right)
$$

for $x_{0} \geqq 0$ is again a solution of $(5 \mathrm{a}, \mathrm{c})$ and is of class $C_{\infty}$. We shall prove that this function $u$ has $x_{0}=0$ as natural boundary.

Since $\eta \neq 0$ there exists a real vector $y$ such that 


$$
y \cdot \eta=2 \pi \text {. }
$$

We have for any positive integer $r$ and for $x_{0} \geqq 0$ from (8)

$$
u\left(x_{0}, x+2^{-r} y\right)-u\left(x_{0}, x\right)=w_{r}\left(x_{0}, x\right),
$$

where

(9a) $w_{r}\left(x_{0}, x\right)=\sum_{j=0}^{r-1} e^{-j^{2}} \exp \left(i 2^{j} \eta \cdot x\right) f\left(2^{j} x_{0}\right)\left(\exp \left(i 2^{j-r+1} \pi\right)-1\right)$

is an entire analytic solution of (5a).

If $u\left(x_{0}, x\right)$ would not have the plane $x_{0}=0$ as natural boundary there would exist a point $x=X$, a positive number $\epsilon$, and a function $U\left(x_{0}, x\right)$ of class $C_{N}$ in

$$
|x-X|<\epsilon, \quad\left|x_{0}\right|<\epsilon
$$

which satisfies (5a) and agrees with $u\left(x_{0}, x\right)$ for $x_{0} \geqq 0$.

The expression

$$
V\left(x_{0}, x\right)=U\left(x_{0}, x+2^{-r} y\right)-U\left(x_{0}, x\right)-w_{r}\left(x_{0}, x\right)
$$

is a solution of (5a) defined for

$$
|x-X|<\epsilon / 2, \quad\left|x_{0}\right|<\epsilon
$$

provided $r$ is so large that

$$
2^{r}>2|y| / \epsilon \text {. }
$$

Moreover $V\left(x_{0}, x\right) \equiv 0$ for $x_{0} \geqq 0$. It follows from the uniqueness theorem of Holmgren ${ }^{4}$ that there exists a positive $\epsilon^{\prime}$, independent of $r$, such that $V\left(x_{0}, x\right)=0$ for all $x_{0}, x$ with

$$
|x-X|<\epsilon^{\prime}, \quad\left|x_{0}\right|<\epsilon^{\prime} .
$$

In other words, the functional equation (9) satisfied by $u$ must be preserved under continuation.

Let $Q\left(\xi_{0}, \xi\right)$ denote the differential operator

$$
Q\left(\xi_{0}, \xi\right)=\prod_{k=2}^{s+1}\left(\xi_{0}-\lambda_{k} \eta \cdot \xi\right)
$$

Since

$$
\prod_{k=2}^{8+1}\left(\xi_{0}-i \lambda_{k}\right) f\left(x_{0}\right)=e^{i \lambda_{1} x_{0}}
$$

\footnotetext{
${ }^{4}$ See John [14].
} 
we have for $|x-X|<\epsilon / 2,-\epsilon<x_{0}<0$

$$
\begin{aligned}
\left|Q\left(\xi_{0}, \xi\right)\left[U\left(x_{0}, x+2^{-r} y\right)-U\left(x_{0}, x\right)\right]\right| \\
=\left|\sum_{j=0}^{r-1} 2^{s i} e^{-j^{2}} \exp \left(i 2^{j} x \cdot \eta\right)\left(\exp \left(i 2^{j-r+1} \pi\right)-1\right) \exp \left(i 2^{i} \lambda_{1} x_{0}\right)\right| \\
\geqq 2 \cdot 2^{s(r-1)} \exp \left(-(r-1)^{2}-2^{r-1} x_{0} \operatorname{Im}\left(\lambda_{1}\right)\right) \\
\quad-2 \exp \left(-2^{r-2} x_{0} \operatorname{Im}\left(\lambda_{1}\right)\right) \sum_{j=0}^{\infty} 2^{s i} e^{-j^{2}} .
\end{aligned}
$$

Hence

$$
\lim _{r \rightarrow \infty}\left|Q\left(\xi_{0}, \xi\right)\left[U\left(x_{0}, x+2^{-r} y\right)-U\left(x_{0}, x\right)\right]\right|=\infty .
$$

But this is incompatible with $U\left(x_{0}, x\right)$ belonging to class $C_{N}$. Consequently $u\left(x_{0}, x\right)$ has the plane $x_{0}=0$ as natural boundary, if we can find a real $\eta$ with $|\eta|=1$ such that the corresponding roots $\lambda_{k}$ of the characteristic equation (6) satisfy $(7 \mathrm{a}, \mathrm{b})$. Since equation (6) is homogeneous in $\eta_{0}$ and $\eta$, the restriction $|\eta|=1$ is unessential.

We call boundary conditions for a solution on the plane $x_{0}=0$ adequate for continuation, if every sufficiently regular solution defined on one side of the plane in a neighborhood of the plane can be continued locally across that plane. We can then formulate our result as follows:

For the boundary conditions (5c) to be adequate for continuation of a solution of (5a) it is necessary that for every real $\eta$ the roots $\lambda_{k}$ are either all real, or that at least $N-s$ of them have positive real part and at least $N-s$ of them have negative real part.

Consequently for $s<N / 2$, the boundary conditions (5c) can be adequate for continuation only if for every real $\eta$ the roots $\lambda_{k}$ are all real, or, in other words, they are never adequate unless the differential equation (5a) is hyperbolic with respect to the plane $x_{0}=0$.

If $N$ is even and $s=N / 2$, conditions (5c) express that $u$ has vanishing Dirichlet data on $x_{0}=0$. Then the vanishing of the Dirichlet data can be adequate for continuation only if for any real $\eta$ the solutions $\eta_{0}$ of (6) are either all real or all imaginary. They are all imaginary when equation (5a) is elliptic. In this case vanishing of the Dirichlet data is adequate for continuation by virtue of the result of Morrey and Nirenberg mentioned above. It is possible that the necessary condition for adequacy of boundary data is always sufficient, regardless of the type of the equation.

We apply the condition to the example of the equation of elastic waves in an istropic medium: 


$$
\left(\frac{\partial^{2}}{\partial t^{2}}-c^{2} \Delta\right)\left(\frac{\partial^{2}}{\partial t^{2}}-c^{\prime 2} \Delta\right) u(x, y, z, t)=0
$$

where $\Delta$ is the Laplace operator in $x y z$-space. Here the boundary conditions

$$
u=\frac{\partial u}{\partial x}=0 \quad \text { for } x=0
$$

are inadequate for continuation, if the constants $c$ and $c^{\prime}$ are distinct. For, in that case, there exist real $y, z, t$ such that the 4 roots of the characteristic equation

$$
\left(t^{2}-c^{2}\left(x^{2}+y^{2}+z^{2}\right)\right)\left(t^{2}-c^{\prime 2}\left(x^{2}+y^{2}+z^{2}\right)\right)=0
$$

are neither all real nor all imaginary. If, on the other hand, $c=c^{\prime}$, the conditions (11b) are adequate; indeed, then, continuation is achieved by the explicit formula (analogous to (3c))

$$
u(-x, y, z, t)=-u+2 x u_{x}+\frac{x^{2}}{c^{2}}\left(\frac{\partial^{2}}{\partial t^{2}}-c^{2} \Delta\right) u
$$

where on the right hand side the arguments of $u$ are $x, y, z, t$.

Equation (11a) is hyperbolic with respect to the plane $t=0$. Thus the boundary conditions

$$
u=u_{t}=0 \quad \text { for } t=0
$$

are adequate for continuation, even when $c \neq c^{\prime}$. Here again an explicit "reflection formula" exists, giving the continuation, which will be derived later (see formula (32c)).

We next turn to the question: Can continuation be achieved by a simple formula of the type (1c) or (3c), if the data are adequate for continuation? Instead of trying to give directly a more precise meaning to what constitutes a "simple formula" it is convenient to take notice of a more qualitative feature of formulae (1c), (3c), namely that they constitute "reflection principles." The values of the continued function at one point $P$ are expressible in terms of the values of the original functions and of its derivatives in points on the line through $P$ perpendicular to the boundary plane. We shall restrict ourselves to the case, where the order $N$ of the differential equation is even, $N=2 m$, and where the boundary conditions on the plane $x_{0}=0$ consist in the vanishing of the Dirichlet data $(s=m)$ :

$$
\left(\xi_{0}^{k} u\right)_{x_{0}=0}=0 \quad \text { for } k=0, \cdots, m-1 .
$$

We are led to the following definition: Equation (5a) is said to 
possess a reflection principle (with respect to the Dirichlet data on the plane $x_{0}=0$ ), if there exists a number $\epsilon>0$ (depending only on $P$ ) such that every solution $u\left(x_{0}, x\right)$ of $(5 \mathrm{a})$, which is analytic in a cylindrical set which has the domain $D$ in $x$-space as base

$$
x \text { in } D, \quad 0 \leqq x_{0}<h
$$

can be continued as a regular analytic solution into the set

$$
x \text { in } D,-\epsilon h<x_{0} \leqq 0,
$$

provided $u$ satisfies the boundary conditions (12) for $x$ in $D$.

It turns out that all equations having a reflection principle in this sense can be characterized rather simply, and that existence of a reflection principle implies existence of an explicit formula for continuation of a solution.

It follows from the definition that an equation (5a) possesses no reflection principle, if there exists an analytic solution $u\left(x_{0}, x\right)$ of (5a) with vanishing Dirichlet data on $x_{0}=0$, which is regular for $x=0, x_{0} \geqq 0$, and has a singularity in some point of the negative $x_{0}$ axis. Let now $\eta$ be any complex $n$-vector $\neq 0$, and let $\lambda_{1}, \cdots, \lambda_{2 m}$ be the corresponding roots $\eta_{0}$ of (6). Then

$$
\begin{aligned}
u\left(x_{0}, x\right) & =\frac{x_{0}^{m}}{\prod_{k=1}^{m+1}\left(\eta \cdot x+\lambda_{k} x_{0}+\lambda_{1}\right)} \\
& =\frac{(-1)^{m+1}}{2 \pi i} \oint_{c} \frac{d \lambda}{\left(\eta \cdot x+\lambda x_{0}+\lambda_{1}\right) \prod_{k=1}^{m+1}\left(\lambda-\lambda_{k}\right)},
\end{aligned}
$$

(where $C$ is a small path about the point $\left.\lambda=-\left(\eta \cdot x+\lambda_{1}\right) / x_{0}\right)$ is easily seen to be an analytic solution of (5a), (12) which is singular at the point

$$
x=0, \quad x_{0}=-1 .
$$

If this solution is regular for $x=0$, and $x_{0} \geqq 0$, there is no reflection principle. This is the case, when

$$
\lambda_{k} x_{0}+\lambda_{1} \neq 0 \text { for real } x_{0} \geqq 0 \text { and } k=1, \cdots, m+1 .
$$

Thus necessary for the existence of a reflection principle is that whenever one root $\lambda_{1}$ is different from 0 then at least one out of any $m$ of 
the remaining $2 m-1$ roots lies on the ray opposite to that containing $\lambda_{1}$. In other words, it is necessary that for any complex $\eta$ either the roots $\lambda_{k}$ of (6) all vanish, or that none of them vanishes and that they all lie on the same line through the origin, half of them on either side of the origin.

We are led to look for the forms $P\left(\eta_{0}, \eta\right)$ whose roots $\eta_{0}=\lambda_{k}$ lie on a straight line through the origin for any complex $\eta$. There would have to exist a real $\theta$ and real $c_{k}$ such that

$$
\lambda_{k}=c_{k} e^{i \theta} \quad \text { for } k=1, \cdots, 2 m .
$$

Let

$$
P\left(\eta_{0}, \eta\right)=\eta_{0}^{2 m}+q_{1}(\eta) \eta_{0}^{2 m-1}+q_{2}(\eta) \eta_{0}^{2 m-2}+\cdots+q_{2 m}(\eta),
$$

where $q_{k}(\eta)$ is a form of degree $k$ in $\eta=\left(\eta_{1}, \cdots, \eta_{n}\right) ; q_{k}(\eta) e^{-i k \theta}$ would have to be real for any $k=1, \cdots, 2 m$. Hence for any complex $\eta$ and any $k, j=1, \cdots, 2 m$

$$
\frac{\left(q_{k}(\eta)\right)^{j}}{\left(q_{j}(\eta)\right)^{k}}
$$

would have to be real, whenever $q_{j}(\eta) \neq 0$. It follows that the expression (14) must be constant, since an analytic function of $\eta$ which is real for all complex $\eta$ in the neighbourhood of a point in $\eta$-space must be a constant.

Let $S$ be the subset of the set of integers $j=1, \cdots, 2 m$, for which $q_{j}(\eta) \not \equiv 0$. Unless $P \equiv \eta_{0}^{2 m}$, the set $S$ is not empty. Let $\mu$ be the greatest common divisor of all the numbers in $S$. We can represent $\mu$ in the form

$$
\mu=\sum_{j \subset S} j n_{j}
$$

with integral coefficients $n_{j}$. Let $Q=Q(\eta)$ be the rational function defined by

$$
Q=\prod_{j \subset S} q_{j}(\eta)^{n_{j}}
$$

Then for any $k$ in $S$

$$
\frac{q_{k}(\eta)^{\mu}}{Q(\eta)^{k}}=\prod_{j \subset S} \frac{q_{k}(\eta)^{j n_{j}}}{q_{j}(\eta)^{k n_{j}}}=\text { constant }=\left(\gamma_{k}\right)^{\mu}
$$

or

$$
q_{k}(\eta)=\gamma_{k}(\eta) Q(\eta)^{k / \mu}
$$

where $\left(\gamma_{k}\right)^{\mu}$ is independent of $\eta$ and $\neq 0$. Since the polynomial $q_{k}(\eta)$ 
is bounded for all bounded $\eta$, the same holds for the rational function $Q(\eta)$, which consequently must be a polynomial in $\eta$. The number $k / \mu$ is an integer for $k$ in $S$, and thus, by (15), $\gamma_{k}$ is independent of $\eta$. It follows that $P$ is of the form

$$
P=\sum_{0 \leqq r \leqq 2 m / \mu} \eta_{0}^{2 m-r \mu} c_{r} Q(\eta)^{r} .
$$

Here the $c_{r}$ are constant, and $Q$ is a form of degree $\mu$. Whenever $\eta_{0}=\lambda$ is a root of the polynomial $P$ given by (16), $\epsilon \lambda$ is also a root, where $\epsilon$ is any $\mu$ th root of unity. For $\lambda \neq 0, \mu>2$, the various $\epsilon \lambda$ do not all lie on a straight line. Hence $P$ is either given by $\eta_{0}^{2 m}$ or by (16), where $Q$ is a form of degree $\mu$ with $\mu=1$ or $\mu=2$. In either case we can factor $P$, and conclude that a reflection principle is possible only if $P$ is of one of the following 3 types:

$$
\begin{aligned}
& P=\eta_{0}^{2 m}, \\
& P=\prod_{k=1}^{2 m}\left(\eta_{0}-a_{k} L(\eta)\right), \\
& P=\prod_{k=1}^{m}\left(\eta_{0}^{2}-a_{k}^{2} q(\eta)\right) .
\end{aligned}
$$

Here the $a_{k}$ are constant, and $L$ and $q$ denote, respectively, a linear or a quadratic form in $\eta$ which is independent of $k$. Since the roots $\eta_{0}$ must either vanish or lie on one line through the origin, half on each side, we can change $L$ or $q$ by a constant factor, so that in (17b) the $a_{k}$ are all real, $a_{1}, \cdots, a_{m}$ positive, $a_{m+1}, \cdots, a_{2 m}$ negative, and in $(17 \mathrm{c})$ so that all the $a_{k}$ are real and positive.

It remains to show that for any equation of one of the types $(17 \mathrm{a}, \mathrm{b}, \mathrm{c})$ with real $a_{k}$ of the correct sign there actually exists a reflection principle. This is achieved by constructing an explicit formula that represents a continuation of the desired type. We omit the case of an equation of type (17a) in which continuation is trivial from further consideration. To arrive at reflection formulae for equations of type $(17 b, c)$, we first derive such a formula for the case of an ordinary differential equation with constant coefficients.

Let $p(\lambda)$ be a polynomial of degree $2 m$ with roots $a_{1}, \cdots, a_{2 m}$, where $a_{k} \neq a_{j}$ for $k=1, \cdots, m$ and $j=m+1, \cdots, 2 m$, and all the $a_{i}$ are different from 0 . We consider a solution $u\left(x_{0}\right)$ of the equation

$$
P\left(\xi_{0}\right) u\left(x_{0}\right)=0
$$

for which

$$
\left(\xi_{0}^{k} u\left(x_{0}\right)\right)_{x_{0}=0}=0 \quad \text { for } k=0,1, \cdots, m-1 .
$$


We put

$$
A(\lambda)=\prod_{k=1}^{m}\left(\lambda-a_{k}\right), \quad B(\lambda)=\sum_{k=m+1}^{2 m}\left(\lambda-a_{k}\right),
$$

so that $p(\lambda)=A(\lambda) B(\lambda)$. Let $C^{\prime}$ and $C^{\prime \prime}$ be two closed paths in the $\lambda$ plane not containing the origin which are exterior to each other, and such that $C^{\prime}$ contains $a_{1}, \cdots, a_{m}$ and $C^{\prime \prime}$ contains $a_{m+1}, \cdots, a_{2 m}$. We define for any $\lambda, \mu$ the polynomial $R(\lambda, \mu)$ by

$$
R(\lambda, \mu)=\frac{p(\lambda)-p(\mu)}{\lambda-\mu} .
$$

Then for any integer $j \geqq 0$ the identity

$$
\begin{aligned}
\xi_{0}^{j} u\left(x_{0}\right)=(2 \pi i)^{-2} \oint_{C^{\prime}} d \alpha \oint_{C^{\prime \prime}} d \beta & \frac{\beta^{i} R\left(\frac{\alpha}{\beta} \xi_{0}, \alpha\right) u\left(\frac{\beta}{\alpha} x_{0}\right)-\alpha^{i} R\left(\frac{\beta}{\alpha} \xi_{0}, \beta\right) u\left(\frac{\alpha}{\beta} x_{0}\right)}{(\beta-\alpha) A(\alpha) B(\beta)}
\end{aligned}
$$

is satisfied for every solution $u$ of $(18 \mathrm{a}, \mathrm{b})$. The expression on the righthand side of (19) is clearly defined since any solution $u$ of (18a) is analytic, and $\left(R(\alpha / \beta) \xi_{0}, \alpha\right)$ is a differential operator depending regularly on the parameters $\alpha, \beta$.

Identity (19) can be verified most easily by observing that the general solution $u$ of $(18 \mathrm{a}, \mathrm{b})$ is of the form

$$
u\left(x_{0}\right)=\frac{1}{2 \pi i} \oint_{c} \frac{h(\lambda) e^{\lambda x_{0}}}{p(\lambda)} d \lambda,
$$

where $h(\lambda)$ is an arbitrary polynomial in $\lambda$ of degree $<m$, and $C$ is a path containing all roots of $p$. We may assume that $C$ contains both $C^{\prime}$ and $C^{\prime \prime}$ in its interior. Then

$$
\begin{aligned}
R\left(\frac{\alpha}{\beta} \xi_{0}, \alpha\right) u\left(\frac{\beta}{\alpha} x_{0}\right) & =\frac{1}{2 \pi i} \oint_{C} \frac{p(\lambda)-p(\alpha)}{(\lambda-\alpha) p(\lambda)} h(\lambda) e^{\beta \lambda x_{0} / \alpha} d \lambda \\
& =h(\alpha) e^{\beta x_{0}}-\frac{p(\alpha)}{2 \pi i} \oint_{C} \frac{h(\lambda)}{(\lambda-\alpha) p(\lambda)} e^{\beta \lambda x_{0} / \alpha} d \lambda
\end{aligned}
$$

The second term makes no contribution, since

$$
\frac{p(\alpha)}{(\beta-\alpha) A(\alpha)(\lambda-\alpha)} e^{\beta \lambda x_{0} / \alpha}
$$


is a regular function of $\alpha$ for $\alpha$ inside $C^{\prime}, \beta$ on $C^{\prime \prime}, \lambda$ on $C$ and the point $\alpha=0$ outside $C^{\prime}$. Moreover, from the theory of residues

$$
\frac{1}{2 \pi i} \oint_{C^{\prime}} \frac{h(\alpha)}{(\beta-\alpha) A(\alpha)} d \alpha=\frac{h(\beta)}{A(\beta)},
$$

since $h$ is of lower degree than $A$, and $\alpha=\beta$ is the only pole of the integrand outside $C^{\prime}$. It follows that

$$
(2 \pi i)^{-2} \oint_{C^{\prime}} d \alpha \oint_{C^{\prime \prime}} d \beta \frac{\beta^{j} R\left(\frac{\alpha}{\beta} \xi_{0}, \alpha\right) u\left(\frac{\beta}{\alpha} x_{0}\right)}{(\beta-\alpha) A(\alpha) B(\beta)}=\frac{1}{2 \pi i} \oint_{C^{\prime \prime}} \frac{\beta^{j} e^{\beta x_{0}} h(\beta)}{p(\beta)} d \beta .
$$

Evaluating the remaining portion of the right hand side of (19), in a similar way, the validity of the identity (19) becomes obvious if we compare it with the expression for $\xi_{0}^{j} u\left(x_{0}\right)$ arising from (20).

In principle the right hand side of identity (19) can be evaluated by the theory of residues. It then yields a representation of $\xi_{0}^{j} u\left(x_{0}\right)$ in terms of $u$ and its derivatives taken for the arguments $a_{k} x_{0} / a_{j}$ and $a_{j} x_{0} / a_{k}$ where $k=1, \cdots, m$ and $j=m+1, \cdots, 2 m$. If all $a_{k}$ are positive all $a_{j}$ negative, this represents a reflection principle, since all $a_{k} / a_{j}$ are then negative and since for $x_{0}<0$ we can express $\xi_{0}^{\jmath} u\left(x_{0}\right)$ in terms of $u$ and its derivatives for positive arguments. If all $a_{k}, a_{j}$ are distinct we have, for example, the formula

$$
\xi_{0}^{j} u\left(x_{0}\right)=\sum_{k=1}^{m} \sum_{r=m+1}^{2 m}
$$

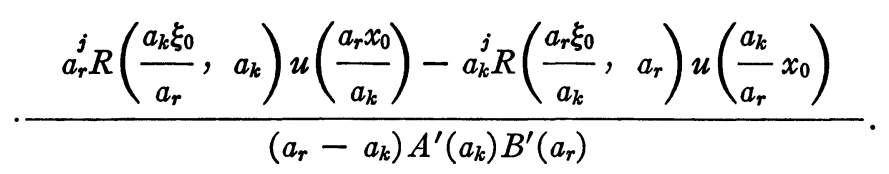

Here

$$
R\left(\frac{a_{k} \xi_{0}}{a_{r}}, a_{k}\right) u\left(\frac{a_{r} x_{0}}{a_{k}}\right)=\left[\frac{p(d / d s)}{d / d s-a_{k}} u(s)\right]_{s=a_{r} x_{0} / a_{k}} .
$$

If we differentiate identity (19) $k$ times with respect to $x_{0}$ and put $x_{0}=0$ we obtain the relation

$$
\left[\xi_{0}^{j+k} u\left(x_{0}\right)\right]_{x_{0}=0}=\left[F_{j k}\left(\frac{d}{d s}\right) u(s)\right]_{\varepsilon=0}
$$

where $F_{j k}(\lambda)$ is a polynomial of degree $\leqq 2 m-1+k$ : 


$$
F_{j k}(\lambda)=(2 \pi i)^{-2} \oint_{C^{\prime}} d \alpha \oint_{C^{\prime \prime}} d \beta \frac{\lambda^{k}\left[\beta^{k+j} \alpha^{-k} R(\lambda, \alpha)-\alpha^{k+j} \beta^{-k} R(\lambda, \beta)\right]}{(\beta-\alpha) A(\alpha) B(\beta)}
$$

There exist polynomials $G_{j k}(\lambda)$ and $H_{j k}(\lambda)$ such that

$$
\lambda^{j+k}=F_{j k}(\lambda)+G_{j k}(\lambda)+H_{j k}(\lambda) p(\lambda)
$$

where $G_{j k}$ is of degree $<2 m$. Then from (21) we have

$$
\left[G_{j k}\left(\xi_{0}\right) u\left(x_{0}\right)\right]_{x_{0}=0}=0
$$

for any solution $u$ of $(18 \mathrm{a}, \mathrm{b})$. Since for such a $u$ the values of $\left[\xi_{0}^{k} u\left(x_{0}\right)\right]_{x_{0}=0}$ vanish for $k=0, \cdots, m-1$ and are arbitrary for $k=m, m+1, \cdots, 2 m-1$, it follows that $G_{j k}$ must be of degree $<m$. Hence there exists an identity of the form (23) with a polynomial $G_{j k}$ of degree $<m$.

We are now in a position to write down a reflection principle for an equation of the type $(17 \mathrm{~b})$. Let $u\left(x_{0}, x\right)$ be a solution of the differential equation

$$
P\left(\xi_{0}, \xi\right) u\left(x_{0}, x\right)=\prod_{k=1}^{2 m}\left(\xi_{0}-a_{k} L(\xi)\right) u\left(x_{0}, x\right)=0
$$

which is analytic for real $x_{0}, x$ in a set (13a), and satisfies the Dirichlet conditions (12) for $x$ in $D$. Here the $a_{k}$ shall be real numbers, $a_{1}, \cdots, a_{m}$ positive, $a_{m+1}, \cdots, a_{2 m}$ negative, and $L$ shall be a linear form with constant (real or complex) coefficients.

Formally the conditions on $u$ are of the type $(18 \mathrm{a}, \mathrm{b})$, only with $a_{k}$ replaced by $a_{k} L(\xi)$. For $j=2 m-1$, we are then led to the identity

$$
\xi_{0}^{2 m-1} u\left(x_{0}, x\right)=v\left(x_{0}, x\right)
$$

where

(26a) $v\left(x_{0}, x\right)=(2 \pi i)^{-2} \oint_{C^{\prime}} d \alpha \oint_{C^{\prime \prime}} d \beta \frac{S}{(\beta-\alpha) A(\alpha) B(\beta)}$,

$$
\begin{gathered}
S=\beta^{2 m-1} \frac{P\left(\frac{\alpha}{\beta} \xi_{0}, \xi\right)-P(\alpha L(\xi), \xi)}{\frac{\alpha}{\beta} \xi_{0}-\alpha L(\xi)} u\left(\frac{\beta}{\alpha} x_{0}, x\right) \\
-\alpha^{2 m-1} \frac{P\left(\frac{\beta}{\alpha} \xi_{0}, \xi\right)-P(\beta L(\xi), \xi)}{\frac{\beta}{\alpha} \xi_{0}-\beta L(\xi)} u\left(\frac{\alpha}{\beta} x_{0}, x\right) .
\end{gathered}
$$


Here the polynomials $A(\alpha), B(\beta)$ are defined by (18c); the path $C^{\prime}$ can be chosen close to the smallest interval on the positive real axis containing the points $a_{1}, \cdots, a_{m}$ and $C^{\prime \prime}$ close to the smallest interval on the negative real axis containing the points $a_{m+1}, \cdots, a_{2 m}$. Then $\alpha / \beta$ and $\beta / \alpha$ are always close to a negative real number whose absolute value does not exceed the largest of the values $-a_{k} / a_{j}$. Let $\epsilon$ be the positive number defined by

$$
\frac{1}{\epsilon}=\underset{k, j=1, \cdots, 2 m}{\operatorname{Max}} \frac{-a_{k}}{a_{j}} .
$$

Then for $\alpha$ on $C^{\prime}, \beta$ in $C^{\prime \prime}$ the quantities $\alpha / \beta$ and $\beta / \alpha$ will be close to negative real numbers lying in the interval from $-1 / \epsilon$ to 0 . Furthermore, for $x$ in $D$ and $-\epsilon h<x_{0} \leqq 0$ the quantities $u\left((\alpha / \beta) x_{0}, x\right)$ and $u\left((\beta / \alpha) x_{0}, x\right)$ will be defined as well as $S$ which represents a differential expression on $u$ and depends regularly on the parameters $\alpha, \beta$. (The corresponding formula for $j<2 m-1$ would still contain the factor $L(\xi)^{i-2 m+1}$, which would not be a differential operator.) It follows that the function $v\left(x_{0}, x\right)$ is defined and analytic for real $x_{0}, x$ in the set (13b). In order to prove that it represents a continuation of $\xi_{0}^{2 m-1} u$ it is sufficient to compare the derivatives with respect to $x_{0}$ at $x_{0}=0$. Comparison with formula (22) shows that this amounts to proving the identity

$$
\left[\xi_{0}^{2 m-1+k} u\left(x_{0}, x\right)\right]_{x_{0}=0}=\left[L^{2 m-1+k}(\xi) F_{2 m-1 k}\left(\frac{1}{L(\xi)} \frac{d}{d s}\right) u(s, x)\right]_{s=0}
$$

for $j=2 m-1, k=0,1,2, \cdots$ and any solution $u$ of (24), (12). By (23) we have

$$
\begin{aligned}
\xi_{0}^{2 m-1+k}= & L^{2 m-1+k} F_{2 m-1 k}\left(L^{-1} \xi_{0}\right)+L^{2 m-1+k} G_{2 m-1 k}\left(L^{-1} \xi_{0}\right) \\
& +L^{k-1} H_{2 m-1 k}\left(L^{-1} \xi_{0}\right) L^{2 m} p\left(L^{-1} \xi_{0}\right)
\end{aligned}
$$

identically in $\xi_{0}$ and $L$. For $L=L(\xi)$ both sides represent differential operators. We apply this identity to a solution of (24), (12) obtaining (27) since

$$
L^{2 m}(\xi) p\left(L^{-1}(\xi) \xi_{0}\right) u\left(x_{0}, x\right)=P\left(\xi_{0}, \xi\right) u\left(x_{0}, x\right)=0
$$

and since also

$$
\left[L^{m-1}(\xi) G_{2 m-1 k}\left(L^{-1}(\xi) \xi_{0}\right) u\left(x_{0}, x\right)\right]_{x_{0}=0}=0,
$$

$L^{m-1}(\xi) G_{2 m-1 k}\left(L^{-1}(\xi) \xi_{0}\right)$ being a polynomial of degree $<m$. This completes the proof of the fact that $v\left(x_{0}, x\right)$ represents an analytic continuation of $\xi_{0}^{2 m-1} u\left(x_{0}, x\right)$. 
The function $v$ is given by a double integral in the complex $\alpha$ - and $\beta$-planes; the integrand has poles at the zeros of $A(\alpha)$ and $B(\beta)$. The integral can be expressed by the residues at these poles, which in turn can be written as differential operators acting on the integrand. In principle then $v\left(x_{0}, x\right)$ can be expressed as a combination of derivatives of $u$ taken at the points

$$
\left(\frac{a_{k}}{a_{j}} x_{0}, x\right) \text { and }\left(\frac{a_{j}}{a_{k}} x_{0}, x\right)
$$

with $k=1, \cdots, m$ and $j=m+1, \cdots, 2 m$. The resulting formula involves only $u$ and a finite number of its derivatives in a number of real points, all lying on the same perpendicular to the plane $x_{0}=0$. In case all the $a_{i}$ are distinct the formula is of the form (20a):

$$
\begin{aligned}
\xi_{0}^{2 m-1} u\left(x_{0}, x\right)= & \sum_{k=1}^{m} \sum_{r=m+1}^{2 m} \frac{S_{k r}}{\left(a_{r}-a_{k}\right) A^{\prime}\left(a_{k}\right) B^{\prime}\left(a_{r}\right)}, \\
S_{k r}= & a_{r}^{2 m-1}\left[\frac{P\left(\frac{d}{d s}, \xi\right)-P\left(a_{k} L(\xi), \xi\right)}{\frac{d}{d s}-a_{k} L(\xi)} u(s, \xi)\right] \\
& -a_{k}^{2 m-1}\left[\frac{P\left(\frac{d}{d s}, \xi\right)-P\left(a_{r} L(\xi), \xi\right)}{\frac{d}{d s}-a_{r} L(\xi)} u(s, \xi)\right]_{s=a_{r} x_{0} / a_{k}}
\end{aligned} .
$$

The identity (25) gives a continuation $v$ of $\xi_{0}^{2 m-1} u$. If we define a function $U\left(x_{0}, x\right)$ in $(13 \mathrm{~b})$ by the conditions

$$
\xi_{0}^{2 m-1} U=v, \quad\left(\xi_{0}^{k} U\right)_{x_{0}=0}=\left(\xi_{0}^{k} u\right)_{x_{0}=0} \quad \text { for } k=0, \cdots, 2 m-2,
$$

$U$ and $u$ will agree with all their derivatives at $x_{0}=0$, and hence $U$ will represent an analytic continuation of $u$. The determination of $U$ in terms of $v$ involves only repeated integration along the line $x_{0}=$ constant. This establishes the existence of a reflection principle in the sense defined here for equations of the type (17b). It is easily seen that the resulting formulae yield a continuation of a solution $u$ of (24), (12) not only when $u$ is analytic, but whenever $u$ is sufficiently often differentiable.

Reflection principles for equations of type (17c) are obtained from the same basic formula (19), taken for $j=2 m-2$. If $p(\lambda)$ is an even polynomial, with roots 


$$
\lambda= \pm a_{k} \quad \text { for } k=1, \cdots, m,
$$

we have $B(\lambda)=(-1)^{m} A(-\lambda)$. Replacing $\beta$ by $-\beta$ in (19) we can choose the same path of integration on $C^{\prime}$ for $\alpha$ and $\beta$. We then arrive at the formula

$$
\begin{aligned}
\xi_{0}^{2 m-2} u\left(x_{0}\right)= & \frac{2(-1)^{m}}{(2 \pi i)^{2}} \oint_{C^{\prime}} d \alpha \oint_{C^{\prime}} d \beta \frac{\alpha \beta^{2 m-2}}{(\alpha+\beta) A(\alpha) A(\beta)} \\
& \frac{p\left(\frac{\alpha}{\beta} \xi_{0}\right)-p(\alpha)}{\frac{\alpha^{2}}{\beta^{2}} \xi_{0}^{2}-\alpha^{2}} u\left(\frac{-\beta}{\alpha} x_{0}\right)
\end{aligned}
$$

Here for positive real $a_{1}, \cdots, a_{m}$ the path $C^{\prime}$ can be taken as a set of small circles about the points $a_{1}, \cdots, a_{m}$.

Let now $u\left(x_{0}, x\right)$ be a function which is analytic in the set (13a), satisfies the differential equation

$$
P\left(\xi_{0}, \xi\right) u=\prod_{k=1}^{m}\left(\xi_{0}^{2}-a_{k}^{2} q(\xi)\right) u=0
$$

and the Dirichlet conditions (12), and let

$$
\begin{aligned}
v\left(x_{0}, x\right) & =\frac{2(-1)^{m}}{(2 \pi i)^{2}} \oint_{C^{\prime}} d \alpha \oint_{C^{\prime}} d \beta \frac{\alpha \beta^{2 m-2} S}{(\alpha+\beta) A(\alpha) A(\beta)} \\
S & =\frac{P\left(\frac{\alpha}{\beta} \xi_{0}, \xi\right)-P\left(\alpha(q(\xi))^{1 / 2}, \xi\right)}{\frac{\alpha^{2}}{\beta^{2}} \xi_{0}^{2}-\alpha^{2} q(\xi)} u\left(\frac{-\beta}{\alpha} x_{0}, x\right)
\end{aligned}
$$

then $v$ is defined and analytic in the set $(13 b)$, where

$$
\epsilon=\min _{k, j=1, \ldots, m} \frac{a_{j}}{a_{k}} .
$$

By the same argument as before, comparing derivatives at $x_{0}=0$, one verifies that

$$
\xi_{0}^{2 m-2} u\left(x_{0}, x\right)=v\left(x_{0}, x\right) .
$$

Repeated integration with respect to $x_{0}$ then furnishes an explicit reflection formula for $u\left(x_{0}, x\right)$ itself.

A typical example is given by the case $m=2$ with distinct $a_{k}$. Let $u\left(x_{0}, x\right)$ be a solution of the equation 


$$
\left(\xi_{0}^{2}-a_{1}^{2} q(\xi)\right)\left(\xi_{0}^{2}-a_{2}^{2} q(\xi)\right) u\left(x_{0}, x\right)=0,
$$

where $q$ is a quadratic form and $a_{1}, a_{2}$ are distinct real positive quantities. Let $u$ satisfy the initial conditions

$$
u=\xi_{0} u=0 \quad \text { for } x_{0}=0 .
$$

The residues at the simple poles of the integrand in the expression for $v$ are easily evaluated, and one finds the formula

$$
\begin{aligned}
\left(a_{1}+a_{2}\right)\left(a_{1}\right. & \left.-a_{2}\right)^{2} \xi_{0}^{2} u\left(x_{0}, x\right) \\
= & {\left[\left(a_{1}+a_{2}\right)\left(\left(a_{1}^{2}+\underset{a_{2}}{2}\right) \frac{\partial^{2}}{\partial s^{2}}-2 a_{1}^{2} a_{2}^{2} q(\xi)\right) u(s, x)\right]_{s=-x_{0}} } \\
& -2 a_{1} a_{2}^{2}\left[\left(\frac{\partial^{2}}{\partial s^{2}}-a_{2}^{2} q(\xi)\right) u\left(s, x_{0}\right)\right]_{s=-a_{2} x_{0} / a_{1}} \\
& -2 a_{1}^{2} a_{2}\left[\left(\frac{\partial^{2}}{\partial s^{2}}-a_{1}^{2} q(\xi)\right) u\left(s, x_{0}\right)\right]_{s=-a_{1} x_{0} / a_{2}}
\end{aligned}
$$

In the case of an equation of the type (32a), for which $a_{1}=a_{2}=1$, one obtains a reflection formula either directly from (31) by evaluating residues at double poles, or from (32c) by passing to the limit with $a_{1}$ and $a_{2}$. The resulting formula is

$$
\begin{aligned}
\xi_{0}^{2} u\left(x_{0}, x\right)=\left[\left(\frac{\partial^{2}}{\partial s^{2}}+2 x_{0} \frac{\partial^{3}}{\partial s^{3}}-x_{0}^{2} \frac{\partial^{4}}{\partial s^{4}}+\right.\right. & 2 q(\xi)-4 x_{0} q(\xi) \frac{\partial}{\partial s} \\
& \left.\left.+x_{0}^{2} q(\xi) \frac{\partial^{2}}{\partial s^{2}}\right) u(s, x)\right]_{s=-x_{0}} .
\end{aligned}
$$

Taking into account the initial conditions $(32 \mathrm{~b})$ we can obtain a simpler formula for $u$ itself, which does not involve any quadratures:

$$
u\left(x_{0}, x\right)=\left[\left(-1-2 x_{0} \frac{\partial}{\partial s}-x_{0}^{2} \frac{\partial^{2}}{\partial s^{2}}+x_{0}^{2} q(\xi)\right) u(s, x)\right]_{s=-x_{0}} .
$$

Formula (33) contains formula (3c) for the bi-harmonic equation as the special case $q=-\xi_{1}^{2}$. Reflection formulae for the more general polyharmonic case can be obtained from (31).

One observes that the reflection formulae for partial differential equations of the types $(17 b, c)$ have been obtained formally from the formula (19) which applies to ordinary differential equations, by treating $P\left(\xi_{0}, \xi\right)$ as an ordinary differential operator in $\xi_{0}$. For general forms $P\left(\xi_{0}, \xi\right)$ the formal expressions have no immediate concrete 
interpretation, since the formal roots $\xi_{0}$ of the equation $P\left(\xi_{0}, \xi\right)=0$ are only algebraic functions of differential operators. For equations of the special type $(17 \mathrm{~b}, \mathrm{c})$ meaningful formulae are obtained, due to the fact that in that case the quotients of roots are ordinary numbers instead of operators.

\section{BIBLIOGRAPHY}

1. S. Bochner, Green's formula and analytic continuation, Ann. of Math. Studies, vol. 33, 1954, pp. 1-14.

2. H. Lewy, $A$ note on harmonic functions and a hydrodynamical application, Proc. Amer. Math. Soc. vol. 3 (1952) pp. 111-113.

3. $\mathrm{R}$. Gerber, Sur une condition de prolongement analytique des fonctions harmoniques, C. R. Acad. Sci. Paris vol. 233 (1955) pp. 1560-1562.

4. L. Nirenberg and C. B. Morrey, On the analyticity of the solutions of linear elliptic systems of partial differential equations, Technical Report No. 5, Department of Mathematics, University of California, Berkeley, 1956.

5. C. B. Morrey, A variational method in the theory of harmonic integrals, OSRTechnical Note 55-108, 1955, pp. 18.

6. H. Lamb, Hydrodynamics (reprinted by Dover Publications, 1945).

7. L. M. Milne-Thomson, Theoretical hydrodynamics, 3d ed., Macmillan, 1955.

8. J. J. Stoker, Surface waves in water of variable depth, Quarterly of Applied Mathematics vol. 5 (1947) pp. 1-54.

9. J. B. Diaz and G. S. S. Ludford, Reflection principles for linear elliptic second order partial differential equations with constant coefficients, Scritti Matematici offerti a Mauro Picone, 1955, pp. 87-95.

10. H. Poritsky, Applications of analytic functions to two-dimensional biharmonic analysis, Trans. Amer. Math. Soc. vol. 59 (1946) pp. 258.

11. R. J. Duffin, Continuation of biharmonic functions by reflection. Duke Math. J. vol. 22 (1955) pp. 313-324.

12. - Analytic continuation in elasticity, Carnegie Institute of Technology, Technical Report No. 23 CIT-ORD-6D-TR23, 1956.

13. A. Huber, The reflection principle for polyharmonic functions, Pacific J. Math. vol. 5 (1955) pp. 433-439.

14. F. John, On linear partial differential equations with analytic coefficients. Unique continuation of data, Communications on Pure and Applied Mathematics vol. 2 (1949) pp. 209-253.

15. H. Lewy, On the boundary behavior of minimal surfaces, Proc. Nat. Acad. Sci. U.S.A. vol. 37 (1951) pp. 103-111.

New York University 